\title{
The Gravitational Field of Light
}

\author{
W. B. BoNNOR \\ Queen Elizabeth College, London \\ Received April 21, 1969
}

\begin{abstract}
I obtain an exact solution of Einstein's equations representing the gravitational field of a steady beam of light. Another exact solution representing two parallel beams shining in the same sense is also given; they do not interact. From a study of null geodesics I conclude that a uniform beam of light is gravitationally stable.

The exact solutions are plane-fronted gravitational waves. It seems that a large class of these waves have as their sources pulses and beams of light.
\end{abstract}

\section{§ 1. Introduction}

Tolman (1934) made an extensive study of the gravitational field of light pulses and beams in the linear approximation to relativity theory. In this paper I give exact solutions corresponding to some of ToLMaN's approximate ones. The exact solutions confirm one of ToLmaN's results, namely that a test ray projected parallel to a steady light beam (and moving in the same sense) is undeflected. Indeed I am able to strengthen this to the statement that two steady parallel light beams (shining in the same sense) do not interact, and hence remain parallel.

Tolman also discussed the stability of a light beam, and I take up this problem by studying the null geodesics within the beam, and conclude that a uniform steady beam is gravitationally stable.

An interesting by-product of the work is that the required exact solutions turn out to be metrics of plane-fronted gravitational waves (Brinkmann, 1923; Robinson, 1956; Helly, 1959; Peres, 1959; TAKeno, 1961 ; KUNDT, 1961 ; EHLERS, and KundT, 1962; Edelen, 1966). Thus we have a physical explanation for a large class of these waves - they are caused by pulses and beams of light.

In $\S 2$ plane-fronted waves and their energy tensor are described, and in $\S 3$ I show how one can construct globally regular solutions representing these waves and their sources. In $\S 4 \mathrm{I}$ specialise to the stationary case and present a detailed comparison with the work of TolmaN: an extraordinary fact becomes apparent here - that the exact and linearised solutions are identical. In $\S 5 \mathrm{I}$ give the exterior and interior field of a uniform steady beam of light, and follow it in $\$ 6$ by the field of two such beams (they do not interact). In $\S 7$ two examples of time-dependent fields are given. $\S 8$ contains a detailed investigation of geodesics in the 12 Commun. math. Phys., Vol. 13 
interior and exterior field of a uniform steady light beam, leading to the conclusion that it is gravitationally stable.

To prevent any confusion I emphasise that I am writing throughout about exact solutions of EINsTEIN's equations, except in $\S 4$ where I refer to ToLmaN's work.

\section{§ 2. Plane-Fronted Gravitational Waves}

I start with the metric for plane-fronted gravitational waves (EHLERS and KuNDT, 1962):

$$
d s^{2}=-d x^{2}-d y^{2}+2 d u d v+2 A d u^{2},
$$

where $-\infty<x, y, u, v<\infty$. This metric is one of a class studied by KERR and ScHILD (1965). The function $A(x, y, u)$ will be taken of differentiability class $C^{1}$, piecewise $C^{3}$, and I shall suppose

$$
A \geqq 0 \text {. }
$$

The coordinates will be numbered

$$
x^{1} \equiv x, \quad x^{2} \equiv y, \quad x^{3} \equiv v, \quad x^{4} \equiv u,
$$

so that $x^{1}$ and $x^{2}$ are space-like, $x^{3}$ null and $x^{4}$ timelike. The metric (2.1) has $\left|g_{i k}\right|=-1$, and

$$
g^{i k}=\left(\begin{array}{rrcc}
-1 & 0 & 0 & 0 \\
0 & -1 & 0 & 0 \\
0 & 0 & -2 \mathrm{~A} & 1 \\
0 & 0 & 1 & 0
\end{array}\right)
$$

so that the coordinate hypersurfaces

$$
x^{4} \equiv u=\text { const }
$$

have normal $n_{i}=(0,0,0,1)$ and are null.

The metric (2.1) has for general A one Killing vector

$$
s^{i}=\sqrt{2} \delta_{3}^{i},
$$

which is null (EHLERs and KUNDT, 1962). (The factor $\sqrt{2}$ is inserted for later convenience).

The Ricci tensor for (2.1) has the value

$$
R^{i k}=-\frac{1}{2}\left(A_{11}+A_{22}\right) s^{i} s^{k},
$$

where $A_{11}=\partial^{2} A / \partial x^{2}, A_{22}=\partial^{2} A / \partial y^{2}$. From the field equations

$$
R^{i k}-\frac{1}{2} g^{i k} R=-8 \pi T^{i k},
$$

we have

$$
T^{i k}=(16 \pi)^{-1}\left(A_{11}+A_{22}\right) s^{i} s^{k},
$$


and, since $s^{i}$ is a null vector, we may interpret this as the energy tensor of a fluid of photons moving with velocity $s^{i}$. The energy density will be taken as

$$
\varrho=\frac{1}{16 \pi}\left(A_{11}+A_{22}\right)
$$

the justification for this will appear in $\S 4$. In empty space A satisfies

The transformation

$$
A_{11}+A_{22}=0 \text {. }
$$

$$
\sqrt{2} u=t-z, \quad \sqrt{2} v=t+z
$$

takes (2.1) into

$$
d s^{2}=\left(-d x^{2}-d y^{2}-d z^{2}+d t^{2}\right)+A(d t-d z)^{2} .
$$

When the metric is used in this form, I shall write

$$
\bar{x}^{1} \equiv x, \quad \bar{x}^{2} \equiv y, \quad \bar{x}^{3} \equiv z, \quad \bar{x}^{4} \equiv t .
$$

In these coordinates

$$
\bar{s}^{i}=(0,0,1,1),
$$

and the energy tensor can be written in the form

$$
-\bar{T}_{3}^{3}=-\bar{T}_{3}^{4}=\bar{T}_{4}^{3}=\bar{T}_{4}^{4}=\varrho
$$

which will be needed later.

\section{§ 3. Globally Regular Solutions}

Any non-uniform solution $A(x, y, u)$ of LAPLACE's equation (2.11) must be singular. Suppose it is of class $C^{3}$ except on the 2 -surface

$$
S: x=x_{0}(u), \quad y=y_{0}(u),
$$

$x_{0}$ and $y_{0}$ being of class $C^{3}$. To obtain a global solution of (2.8) we surround $S$ by the 3-surface

$$
\Sigma:\left(x-x_{0}(u)\right)^{2}+\left(y-y_{0}(u)\right)^{2}=\beta^{2}(>0),
$$

and replace $A$ by some function $A^{*}(x, y, u)$ of class $C^{3}$ inside and on $\Sigma$, that is in

$$
D:\left\{(x, y, u, v):\left(x-x_{0}(u)\right)^{2}+\left(y-y_{0}(u)\right)^{2} \leqq \beta^{2}\right\} .
$$

$A^{*}$ has to satisfy

$$
A=A^{*}, \frac{\partial A}{\partial x^{i}}=\frac{\partial A^{*}}{\partial x^{i}}, \text { on } \Sigma .
$$

It is fairly obvious that $A^{*}$ always exists. In the Appendix this is proved rigorously in the case where $A$ is class $C^{4}$ on and near $\Sigma$. The method of proof is to exhibit a function $A^{*}$ satisfying the conditions. (Naturally $A^{*}$ is not unique).

$A^{*}$ will not satisfy (2.11) throughout $D$. Where it does not, $T^{i k}$ is given by (2.9). In this way we can construct a globally regular solution 
of (2.8) representing light beams or pulses moving with velocity $s^{i}$ within $D$, and empty space outside $D$.

Evidently the procedure can be extended to any finite number of singularities such as (3.1). Singularities on 3 -surfaces $f(x, y, u)=0$ can be treated similarly provided their sections by every hypersurface $u=$ const are finite. I conclude that, confining ourselves to those planefronted gravitational waves whose singularities lie in the finite part of the $(x, y)$ plane, we can by this procedure provide a large class of them with interior solutions and so make them globally regular. It thus seems that this class of plane-fronted gravitational waves have for their sources pulses and beams of light. Further justification from the linearised Einstein equations will be given in $\S 5$.

It turns out that the solutions of most physical interest have, in addition to a singularity on (3.1), a logarithmic singularity at $x=\infty$, $y=\infty$. Reasons will be given later why this is purely a coordinate singularity, of no physical importance. Therefore I shall not hesitate to use these solutions.

\section{\$4. The Stationary Case}

In $\S \S 4-6$ I shall assume

$$
A \equiv A(x, y)
$$

so the metrics (2.1) and (2.13) are stationary. There are now two Killing vectors if $A$ is a general function of $y$ and $x$,

$$
s^{i}=\sqrt{2} \delta_{3}^{i}, \quad \text { and } \quad t^{i}=\delta_{4}^{i},
$$

respectively null and time-like. The energy tensor is now independent of $u$, and, from the argument of $\S 3 \mathrm{I}$ claim that the sources in the finite part of the $(x, y)$ plane are steady light beams parallel to $O z$.

This statement becomes very plausible if, following Tolman (1934), we study the corresponding linear approximation. Consider in special relativity a directed flow of electromagnetic radiation parallel to the positive $z$-direction. The non-zero components of $T_{k}^{i}$ are

$$
-T_{3}^{3}=-T_{3}^{4}=T_{4}^{3}=T_{4}^{4}=\varrho,
$$

where $\varrho$ is the energy density, and we now obtain in the usual way solutions of the linearised Einstein equations corresponding to this $T_{k}^{i}$. Indeed if we write

$$
g_{i k}=\eta_{i k}+\gamma_{i k},
$$

$\eta_{i k}$ being the Minkowski metric components, and $\gamma_{i k}$ being small, we easily find for the non-zero $\gamma_{i k}$

$$
\gamma_{33}=\gamma_{44}=-\gamma_{34}=-4 \int \frac{[\varrho]}{R} d v \stackrel{\text { def }}{=} h,
$$




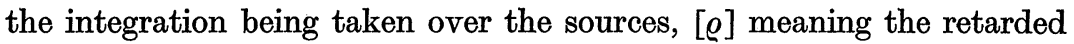
value of $\varrho$, and $R$ being the distance from the source-point to the fieldpoint. If the sources are independent of $z$ and $t$ we have the linearised gravitational field of steady beams of light.

We now compare the approximate solution of the previous paragraph with exact solution of $\S 2$, restricted by (4.1). It turns out that the approximate metric (4.4) and the exact metric (2.13) are identical if we write

$$
h=+A \text {. }
$$

Moreover, if $h$ is independent of $z$ and $t$ it satisfies

$$
h_{11}+h_{22}=16 \pi \varrho,
$$

$\varrho$ being given by (4.3); now $A$ satisfies the same equation. namely (2.10), and the $\varrho$ occuring in the latter is connected with the energy tensor $\bar{T}_{k}^{i}$ by (2.16), which is the same as (4.3). Hence the exact solution and the solutions of the linear approximation are formally identical, and I shall therefore suppose that the former, like the latter, refer to the gravitational field of steady light beams shining in the positive z-direction. The fact that the exact and linearised solutions are identical is highly unusual, but to be expected because the right-hand side of (2.7) contains no non-linear terms.

If the beams shine in the negative $z$-direction the affects are the following. In the linear approximation $T_{3}^{4}, T_{4}^{3}, \gamma_{34}, \gamma_{43}$ change sign and the remaining quantities are unaltered. In the exact solution $\bar{T}_{3}^{4}$ and $\bar{T}_{4}^{3}$ change sign, and (2.13) becomes

$$
d s^{2}=\left(-d x^{2}-d y^{2}-d z^{2}+d t^{2}\right)+A(d t+d z)^{2} .
$$

If the latter metric is submitted to the transformation (2.12) we obtain (2.1) with $u$ and $v$ interchanged. The conclusion of this paragraph is that (2.1) or (2.13) can represent the field of several beams only if they all shine in the positive $z$-direction.

\section{§5. The Field of a Steady Uniform Beam of Circular Cross Section}

Let us now consider a single beam symmetrical about $0 z$ and shining in the positive $z$-direction. For the exterior solution we may take

$$
A_{e}=8 m \log (r / \alpha), \quad r=+\left(x^{2}+y^{2}\right)^{1} / 2,
$$

where $\alpha$ is a positive constant, and $m$ is the constant energy density per unit proper length. The factor 8 is chosen to agree with the expression for $h$ obtained by integrating (4.5) for an infinite line source of constant density $\varrho$, and using (4.6). We notice that the gravitational potential is twice as great as that for a material rod of line density $m$; 
factors of two often occur in relating electromagnetic to mechanical energy. (BonNor, 1960; Tolman, 1934).

We can easily construct an interior solution. Take

$$
A_{i}=4 \pi \varrho_{0} r^{2}, \quad\left(\varrho_{0} \text { const }\right)
$$

which, according to $(2.10)$ and $(2.16)$ corresponds to an energy tensor

$$
-\bar{T}_{3}^{3}=-\bar{T}_{3}^{4}=\bar{T}_{4}^{3}=\bar{T}_{4}^{4}=\varrho_{0}
$$

in the coordinates of (2.13). We may now use (5.1) and (5.2) as the complete solution in conjunction with metric (2.13) or (2.1). Continuity of the $g_{i k}$ and their first derivatives is ensured on the boundary 3surface $r=a$ (const) if we take

$$
m=\pi \varrho_{0} a^{2}, \quad \log a / \alpha=1 / 2 .
$$

These are sufficient to satisfy the standard boundary conditions of general relativity. Thus the solution for a steady uniform beam with circular cross section shining in the positive $z$ direction is (2.13) (or (2.1)) with

$$
\begin{array}{ll}
A_{e}=4 m+8 m \log r / a, & r \geqq a, \\
A_{i}=4 m r^{2} / a^{2}, & r \leqq a .
\end{array}
$$

The logarithmic infinity as $r \rightarrow \infty$ is a coordinate singularity; the paths of test particles and light rays are well-behaved for arbitrary $r$ (see $\S 8$ ), and at any point one may introduce natural (i.e. freely-falling) coordinates in which the metric is locally non singular. Hence the solution is free of singularities.

\section{§ 6. The Field of Two Parallel Beams}

We can satisfy (2.11) by taking

where

$$
A=8 m_{1} \log \left(r_{1} / \alpha_{1}\right)+8 m_{2} \log \left(r_{2} / \alpha_{2}\right),
$$

$$
\begin{aligned}
& r_{1}^{2}=\left(x-a_{1}\right)^{2}+\left(y-b_{1}\right)^{2}, \\
& r_{2}^{2}=\left(x-a_{2}\right)^{2}+\left(y-b_{2}\right)^{2},
\end{aligned}
$$

$m_{i}, \alpha_{i}, a_{i}$ and $b_{i}$ being constants. This represents the exterior field of two beams of light with energy densities $m_{1}$ and $m_{2}$ per unit proper length both shining in the positive $z$-direction.

It is remarkable that one may superpose solutions as in (6.1), without introducing additional terms into the metric. (The situation is entirely different in the Weyl static axially symmetric solutions: in these, if one wishes, for instance, to superpose the solutions for two particles, one has to introduce an additional singular term into the metric representing a strut holding the particles apart). The physical meaning is that the light 
beams do not interact. This too agrees with the result of Touman (1934) in the linear approximation.

Owing to the linearity of (2.10) it is a trivial problem to construct interior solutions for both beams.

\section{§ 7. Two Time-Dependent Fields}

I give two examples of time-dependent fields.

The space-time generated by

$$
A=\phi(u) \log (r / \alpha), \quad \alpha=\text { const , }
$$

represents a beam of light travelling along the $z$-axis, and of variable intensity unless $\phi$ is constant. An interior solution can be obtained by the method of $\S 3$ if $\phi$ is of class $C^{3}$. If for example $\phi=\exp \left\{-k^{2} u^{2}\right\}$ we have the field of a pulse of light. Suspending our differentiability requirements and allowing $\phi(u)=\delta(u)$, thereby taking a sharper pulse, we see that the gravitational field precisely keeps pace with the pulse, being zero at every spatial point $(x, y, z)$ except at one instant $z=t$, when it is equal to that of an infinite rod.

The second field comes by taking

$$
A=\phi(u) \log \sqrt{\left(x-x_{0}(u)\right)^{2}+\left(y-y_{0}(u)\right)^{2}} .
$$

This can be thought of as the exterior field of beams from a searchlight at $z=-\infty$ whose position is

$$
x=x_{0}(u), \quad y=y_{0}(u),
$$

but which always shines in the positive $z$-direction, though with variable intensity.

\section{\$ 8. Geodesies and Null Geodesics}

The only non-zero Christoffel symbols for metric (2.1) are

$$
\Gamma_{44}^{1}=A_{1}, \quad \Gamma_{44}^{2}=A_{2}, \quad \Gamma_{14}^{3}=A_{1}, \quad \Gamma_{24}^{3}=A_{2}, \quad \Gamma_{44}^{3}=A_{4} .
$$

The geodesic equations are therefore

$$
\begin{gathered}
\frac{d^{2} x}{d w^{2}}+A_{1}\left(\frac{d u}{d w}\right)^{2}=0, \frac{d^{2} y}{d w^{2}}+A_{2}\left(\frac{d u}{d w}\right)^{2}=0 \\
\frac{d^{2} v}{d w^{2}}+2 A_{1} \frac{d u}{d w} \frac{d x}{d w}+2 A_{2} \frac{d u}{d w} \frac{d y}{d w}+A_{4}\left(\frac{d u}{d w}\right)^{2}=0, \\
\frac{d^{2} u}{d w^{2}}=0,
\end{gathered}
$$

where $w$ is a parameter which is equal to the arc length $s$ in the case of geodesics.

First, we notice that the null line

$$
x=x_{0}, \quad y=y_{0}, \quad v=w+v_{0}, \quad u=u_{0},
$$


where $x_{0}, y_{0}, v_{0}, u_{0}$ are constants, is a null geodesic in the general planefronted wave metric (2.1). The interpretation of this is that no matter what the sources of the field, a light ray moving parallel to $0 z$ and in the positive sense is undeflected. This fact is consistent with the supposition that the sources of (2.1) can be light pulses, moving parallel to the positive sense of $0 z$, and not interacting with one another. In particular, it is of course consistent with our solution (6.1) for non-interacting parallel beams. The null Killing vector $s^{i}$ given in (2.6) is tangent to (8.5) at every point.

For general $A(x, y, u)$, the null geodesic (8.5) is the only one through $\left(x_{0}, y_{0}, v_{0}, u_{0}\right)$ which is undeflected. Any other has $d u / d w \neq 0$ so from (8.2) it will be deflected unless

$$
A_{1}=A_{2}=0 \quad \text { on the null geodesic. }
$$

In particular if we consider the null curve $C$ parallel to the negative $z$-direction in (2.13), its tangent vector is proportional to

$$
p^{i}=(0,0,-A, 1)
$$

in the coordinates of (2.1). This is not a null geodesic unless (8.6) is satisfied. As an example where $C$ is a null geodesic we may take the empty space-time (2.1) with

$$
A=\sin x \cosh y,
$$

which satisfies (2.11). The null curve (8.5) is of course a null geodesic in this space-time; but so is the null curve

$$
x=\frac{1}{2} \pi, \quad y=0, \quad v=-w+v_{0}, \quad u=w+u_{0},
$$

which is parallel to the negative $z$-axis in (2.13). Thus there are special points in some space-times through which rays parallel to $0 z$ in either sense are undeflected; but generally rays in the negative sense are deflected.

Let us now apply the specialisation (4.1) thereby making the field stationary. It is convenient to introduce plane polar coordinates by

$$
x=r \cos \theta, \quad y=r \sin \theta ;
$$

Eqs. (8.2)-(8.4) then become

$$
\begin{gathered}
\ddot{r}-r \dot{\theta}^{2}=-k^{2} \partial A / d r, \\
\frac{1}{r} \frac{d}{d w}\left(r^{2} \dot{\theta}\right)=-\frac{k^{2}}{r} \frac{\partial A}{\partial \theta}, \\
\ddot{v}=-2 k A, \\
\dot{u}=k,
\end{gathered}
$$


where means differentiation with respect to $w$ and $k$ is a constant of integration. Eqs. (8.11) and (8.12) are formally the same as the Newtonian equations of motion of a particle in a plane under a gravitational potential $k^{2} A(r, \theta)$. The right-hand side of (8.13) represents a Coriolis force giving the particle or ray an acceleration in the $z$-direction if it has a velocity in the $x$ or $y$-directions. This is to be expected because the field is stationary but not static.

We now specialise still further by assuming

$$
A=A(r),
$$

as we are going to consider the geodesics of the solution in $\S 5$. From (8.11) [with (8.12)] and (8.13) we find, using the metric form (2.1) to relate arbitrary constants

$$
\begin{aligned}
\dot{r}^{2} & =-h^{2} r^{-2}-2 k^{2} A+2 k c-\sigma, \\
\dot{v} & =-2 k A+c, \\
\dot{\theta} & =h r^{-2},
\end{aligned}
$$

where $A$ is given by (5.5) or (5.6), $c$ and $h$ are further arbitrary constants, and $\sigma$ is zero for a null geodesic and +1 for a non-null geodesic. The case $k=0$ gives rise only to the null geodesic (8.5), so we henceforth suppose that $k \neq 0$. We consider in turn the exterior and interior fields of the light beam described in $\S 5$.

Exterior Field. A is given by (5.5). We can make a number of deductions from the geodesic equations.

(i) No light ray [except (8.5)] and no particle can escape to $r=\infty$. This is clear from (8.16) since, because of the form of $A$, the right-hand side becomes negative as $r \rightarrow \infty$.

(ii) Excepting once again the ray (8.5), every particle or ray projected in the 3 -space $\theta=$ const. eventually hits the light beam. This follows because (8.11) with $\dot{\theta}=0$ shows that $r$ cannot have a minimum or tend to a finite limit as $w \rightarrow \infty$; on the other hand we have just seen that $r$ is bounded, so the result follows.

(iii) If $h \neq 0$ there exist rays and trajectories of test particles which do not reach $r=\infty$, or hit the beam. This is clear because of the presence of the term $-h^{2} r^{-2}$ in (8.16).

(iv) Observationally, the effects discussed here are negligible. Consider, for instance, the case of a ray of light propagated radially outwards in the field of (5.5). Using the metric in form (2.13) with initial conditions

$$
\sigma=0, \quad w=0, \quad r=r_{0}, \quad \theta=\theta_{0}, \quad z=z_{0}, \quad \frac{d z}{d w}=\frac{d \theta}{d w}=0,
$$

we have from (8.14), (8.17) and (8.18), with the use of (2.12)

$$
\dot{v}_{0}=\dot{u}_{0}=k=c-2 k\left(4 m+8 m \log r_{0} / a\right) ; h=0,
$$


whence substituting in (8.16)

$$
\dot{\gamma}^{2}=2 k^{2}\left(1+2 A_{0}-A\right) .
$$

Hence the maximum value of $r$ reached by the ray is given by

whence

$$
A=1+2 A_{0}
$$

$$
r_{\max }=\frac{r_{0}^{2}}{a} \exp \left(\frac{1}{2}+\frac{1}{8 m}\right) \text {. }
$$

The dimensionless quantity $m\left(G d / c^{2}\right.$ where $d$ is the mass per unit length in units of customary dimensions) is extremely small for beams realised in nature. For example considering the light from the Sun which falls on the Earth to constitute a beam, we have $m \sim 6 \times 10^{-8} \mathrm{gm} \mathrm{cm}^{-1}$. Hence $r / r_{0}$ is for practical purposes infinite, and the fact that the ray cannot escape the beam is of no more than theoretical importance.

Interior Field. A is given by (5.6). The geodesic equations can be completely integrated in terms of elementary functions. In particular (8.16) and (8.17) give

$$
\begin{gathered}
r^{2}=\mu+\left(\mu^{2}-\frac{4 h^{2}}{\beta^{2}}\right)^{1 / 2} \sin (\beta w+\varepsilon) \\
v=e+\frac{\sigma w}{2 k}+\frac{\beta}{4 k}\left(\mu^{2}-\frac{4 h^{2}}{\beta^{2}}\right)^{1 / 2} \cos (\beta w+\varepsilon)
\end{gathered}
$$

where

$$
\mu=\frac{(2 k c-\sigma) a^{2}}{16 m k^{2}}>0, \quad \beta^{2}=\frac{32 k^{2} m}{a^{2}},
$$

and $e, \varepsilon$ are additional arbitrary constants. The beam itself is composed of rays such as (8.5), which is of course also a solution of the null geodesic equations. We conclude that if, through a perturbation, one of these rays should assume the form given by (8.23), (8.24) and (8.18), it would oscillate about $r=$ const. Therefore the uniform beam is gravitationally stable ${ }^{1}$.

\section{§9. Conclusion}

The main conclusions of this work are the following.

(i) The gravitational field of light is twice that of a material source of the same energy density.

(ii) The gravitational field of pulses and beams of light consists of plane-fronted gravitational waves, well known already. There is no evidence from my solutions that these waves carry energy away from

1 If the maximum value of $r^{2}$ in (8.23) were greater than $a^{2}$ the ray would leave the beam. It would then follow the path of a null geodesic in the exterior field, and might or might not return to the beam, according to the previous discussion. 
the light pulses and beams which are their sources: the mass parameters of the latter remain unchanged.

(iii) Parallel beams (or pulses) of light shining in the same sense do not interact.

(iv) A uniform beam of light is gravitationally stable.

(v) The new effects studied here are too small for observational detection at present.

After this paper had been submitted for publication Mr. D. RAwson HARRIs called my attention to am interesting paper by Peres (1960) in which the exterior field of $\S 5$ is proposed for that of a light beam, and the solution of $\S 6$ is suggested for two light beams. These matters were however only briefly discussed by Peres, whose interest was different from my own here.

\section{Appendix}

In this Appendix we assume that $A(x, y, u)$ is singular on the 2 -surface (3.1), is of class at least $C^{3}$ elsewhere, and is of class $C^{4}$ on and near $\Sigma$ given by (3.2).

We have to prove existence of a function $A^{*}(x, y, u)$ of class $C^{3}$ in $D$ (3.3), and satisfying (3.4). Take $\beta>0$ and define $G(r, \theta, u)=A(x, y, u)$ where

and also

$$
x-x_{0}(u)=r \cos \theta, \quad y-y_{0}(u)=r \sin \theta ;
$$

$$
\begin{gathered}
f(\theta, u)=G(\beta, \theta, u), \\
g(\theta, u)=\frac{\partial G}{\partial r}(\beta, \theta, u)
\end{gathered}
$$

$f$ and $g$ are of class $C^{3}$ at least because of the hypotheses on $A$.

We next define, on $0 \leqq r \leqq \beta$,

$$
F(r, \theta, u)=\left(\frac{r}{\beta}\right)^{4}\left\{f(\theta, u)-(\beta-r)\left[g(\theta, u)-\frac{4}{\beta} f(\theta, u)\right]\right\} .
$$

Then $F$ is of class $C^{3}$ in all its arguments, and also

$$
F(\beta, \theta, u)=f, \frac{\partial F}{\partial r}(\beta, \theta, u)=g
$$

We now obtain $A^{*}(x, y, u)$ by means of

$$
A^{*}(x, y, u)=F(r, \theta, u) \text {. }
$$

Then $A^{*}$ has the desired properties; in particular it is of class $C^{3}$ at $x=x_{0}, y=y_{0}$, the factor $(r / \beta)^{4}$ having been inserted to ensure this.

\section{References}

Bonnor, W. B.: Proc. Phys. Soc. 76, 899 (1960).

Brinkmann, H. W.: Proc. Natl. Acad. Sci. U.S., 9, 1 (1923).

EdeLeN, D. G. B.: J. Math. Mech., 16, 453 (1966). 
EHLERS, J., and W. KUNDT: Article in: Gravitation an introduction to current research, ed. L. Witten, p. 85, New York: John Wiley 1962.

HeLY, J.: Comp. Rend., 249, 1867 (1959).

Kerr, R. P., and A. Schild: IV Centenario della nascita die Galileo Galilei, Atti del convegno sullarrelatività generale, p. 222, Florence, 1965.

Kundt, W.: Z. f. Phys. 163, 77 (1961).

Peres, A.: Phys. Rev. Letters 3, 571 (1959).

Peres, A.: Phys. Rev. 118, 1105 (1960).

RoBInson, I.: unpublished 1956.

Takmo, H.: Scientific Reports of the Research Institute for Theoretical Physics, Hiroshima, No. 1, (1961).

Tolman, R. C.: Relativity, thermodynamics and cosmology, p. 272 et seq. Oxford: Clarendon Press 1934.

W. B. BonNoR

Department of Mathematics Queen Elizabeth College

Campden Hill Road

London, W. 8, Great Britain 